\title{
Erratum to: Environments in the Outer Solar System
}

\author{
N. Krupp • K.K. Khurana • L. Iess • V. Lainey • \\ T.A. Cassidy • M. Burger · C. Sotin · F. Neubauer
}

Published online: 30 June 2010

(C) Springer Science+Business Media B.V. 2010

\section{Erratum to: Space Sci Rev \\ DOI 10.1007/s11214-010-9653-z}

Two references were erroneously presented as:

J. Jia, R.J. Walker, M.G. Kivelson, K.K. Khurana, J.A. Linker, J. Geophys. Res. 113 (2008). doi: 10.1029/JA012748

The online version of the original article can be found under doi:10.1007/s11214-010-9653-z.

\footnotetext{
N. Krupp (凶)

Max-Planck-Institut für Sonnensystemforschung, Katlenburg-Lindau, Germany

e-mail: krupp@mps.mpg.de
}

K.K. Khurana

IGPP, University of California Los Angeles, Los Angeles, CA, USA

L. Iess

Dipartimento di Ingegneria Aerospaziale ed Astronautica, Universita La Sapienza, Rome, Italy

V. Lainey

IMCCE-Observatoire de Paris, Paris, France

T.A. Cassidy

University of Virgina, Charlottesville, VA, USA

M. Burger

Goddard Space Flight Center, Greenbelt, MD, USA

C. Sotin

Jet Propulsion Laboratory, Pasadena, CA, USA

F. Neubauer

University of Cologne, Cologne, Germany 
J. Jia, R.J. Walker, M.G. Kivelson, K.K. Khurana, J.A. Linker, J. Geophys. Res. 114 (2009). doi: 10.1029/JA014375

The correct presentation of these references is:

X. Jia, R.J. Walker, M.G. Kivelson, K.K. Khurana, J.A. Linker, J. Geophys. Res. 113 (2008). doi: 10.1029/2007JA012748

X. Jia, R.J. Walker, M.G. Kivelson, K.K. Khurana, J.A. Linker, J. Geophys. Res. 114 (2009). doi: 10.1029/2009JA014375 